\title{
The Management of Chronic Venous Ulcers and the Benefit of Subfascial Endoscopic Perforator Vein Surgery
}

\author{
MODERATORS: MURRAY ASCH, M.D., FRCPC, and \\ PETER G. KALMAN, M.D. \\ PANELISTS: $\quad$ GREGORY L. MONETA, M.D. \\ PETER GLOVICZKI, M.D.
}

ABSTRACT Elastic compression is the primary treatment modality of venous ulcers. Local ulcer treatment is important to enhance granulation and prevent or treat infection, while surgical treatment is aimed at correcting the underlying venous stasis and ambulatory venous hypertension. This debate reviews the pros and cons of surgical treatment for venous incompetence and discusses current evidence to support the use of subfascial endoscopic perforator vein surgery (SEPS). Ablation of superficial reflux with high ligation and stripping of the incompetent saphenous vein, done together with avulsion of varicose veins, remains the main surgical treatment of chronic venous insufficiency. Level 1 evidence of clinical and hemodynamic improvement directly related to interruption of incompetent perforators is currently not available. The North American SEPS registry experience and reports from larger centers, however, provide data on rapid ulcer healing, low morbidity, and decreased wound complications from endoscopic perforator interruption. SEPS, together with ablation of the superficial reflux, when present, can be offered to surgical candidates with advanced chronic venous insufficiency and venous ulcers. While improvement in post-thrombotic patients may be modest, those with primary

M.A., Assistant Professor, Vascular and Interventional Radiology, Mount Sinai Hospital and University Health Network, University of Toronto, Ontario, Canada; P.G.K., Vascular Surgeon, Toronto East General Hospital, Division of Vascular Surgery; Associate Professor of Surgery, The University of Toronto, Toronto, Ontario, Canada; G.L.M., Professor of Surgery; Head, Division of Vascular Surgery, Department of Surgery, Oregon Health Sciences University, Portland, OR; P.G., Professor of Surgery, Mayo Medical School; Chair, Division of Vascular Surgery, Mayo Clinic, Rochester, MN.

Copyright (C) 2000 by Thieme Medical Publishers, Inc., 333 Seventh Avenue, New York, NY 10001, USA.

Tel.: +1(212) 584-4662. 0894-8046,p;2000,13,2,103,118,ftx,en; pvs000098 
valvular incompetence will have a predictably good clinical outcome and improved hemodynamic results. The superiority of surgical treatment over optimal medical management remains to be established in a prospective randomized, multi-center trial. The North American Venous Ulcer Surgery (NAVUS) Trial has been designed to answer the role of ablation of the superficial reflux, with or without SEPS, in the treatment of venous ulcers.

\section{Keywords Venous ulcers, surgery, perforating veins, endoscopy SEPS}

Dr. Kalman: I am pleased to begin the first of two debates this afternoon. The first debate focuses around the issues regarding chronic venous stasis ulcers. That is, whether to follow a conservative approach, or consider a minimally invasive surgical approach. We have two individuals who are well versed and respected in the specialty of venous surgery to debate these opposing views. I would like to introduce my co-moderator, Murray Asch, an interventional radiologist who will introduce the first speaker.

Dr. Asch: Thank you Peter, again for organizing such a great meeting and for including me. It is really nice that he does recognize both at work and after work, the importance of radiologists. To begin I thought we would have a vote, take a poll before the first debate. How many here favor a conservative approach for the treatment of chronic venous ulcer disease?

We are now going to count. And now for the surgical approach. It is a clear majority, so now we will have to see what happens. The first speaker is Dr. Greg Moneta, who is the Professor of Surgery at the Oregon Health Sciences University in Portland, Oregon. He has a strong background in venous disease management and is President-Elect of the American Venous Forum. Today he has been asked to take the side of conservative management of chronic venous ulcer disease.

Dr. Moneta: The show of hands indicates conservative therapy is clearly favored by this audience. Even though Dr. Gloviczki is an excellent speaker, it appears that in this case he will have his work cut out for him. Hopefully it will be difficult to convince this savvy audience that an unproven procedure such as subfascial endoscopic perforator surgery (SEPS) should be adapted as a mainstay of treatment for venous ulceration. In this debate we will focus on the role of the SEPS versus conservative treatment of venous ulcer. I will demonstrate that conservative treatment of venous ulceration is at least as good as SEPS.

One of the reasons I am asked to talk about conservative treatment of venous ulcer and argue against the SEPS procedure is that being from Oregon you are supposed to be "against" everything. At Oregon, as you may remember, we were supposed to have been "against" in situ vein grafting. At one time most thought it was going to be superior to reverse vein grafting. We were "against" performing extensive cardiac evaluations on every vascular surgical patient. We were also "against" thrombolysis as effective therapy 
for thrombosed vein grafts. However, as it turns out, in situ and reverse vein grafts work about the same. Dipyridamole thallium scans don't reduce myocardial infarctions associated with vascular surgery, and thrombolysis of thrombosed vein grafts gives poor short- and long-term results. In reality, at Oregon we are not against new operations, new therapies, or new ideas. We are in favor of properly designed and carefully conducted clinical research and evidence-based medicine. I personally hope SEPS will prove to be an effective therapy for venous ulcer; however, careful analysis suggests it is no better than traditional compressive therapy. While this is a procedure that merits continued investigation, the evidence currently available does not suggest that SEPS should be routinely applied in clinical practice.

This slide depicts a venous ulcer. I bet, Peter, you see a few of these venous ulcers at the Mayo Clinic. We certainly see a number of them in Portland. We have noted over the years, and everyone in the audience knows, that if you put these people to bed and elevate the limb, the ulcer will virtually always heal. Unfortunately, most patients cannot be put to bed for an extended period. Therefore, the mainstay of treatment for venous ulcer is ambulatory compressive therapy. I do not believe that it greatly matters how compression is achieved; elastic stockings, various types of wraps, and Unna boots, all work. Compressive therapy, no matter in what form, is highly effective in many physicians' experience in healing venous ulcers.

Compressive therapy is, in fact, the gold standard for treatment of venous ulcer. It has not been validated in a randomized trial versus no therapy, but I think everybody would agree that it works better than nothing.

Operative therapy for venous ulcer has been around for over 80 years, but operative therapy is still not accepted as standard therapy for venous ulceration. Why? Well, SEPS uses all kinds of sophisticated equipment and is a modern, sexy operation. However, there is no evidence it works any better than a standard compressive bandage. SEPS is clearly an evolving therapy. It is evolving in terms of what patients, if any, should be operated, how the procedure is performed, and what adjunctive procedures are performed in addition to SEPS. However, it is important to keep in mind that an evolving therapy does not mean an efficacious therapy. I will agree SEPS is feasible; there is no question you can do it. I agree it is safe. However, there is no good evidence that it is effective.

When we see patients with a venous ulcer, we first evaluate for the extent of associated edema, the presence of stasis dermatitis, and infection. If necessary, edema is initially controlled with a period of bed rest. When present, stasis dermatitis is treated with steroid creams applied to the area of stasis dermatitis, but not to the ulcer itself. Antibiotics are utilized for invasive infection. The ulcer itself is treated with simple saline, wet-to-dry dressings. Compression is provided with 30 to $40-\mathrm{mm}$ elastic compression stockings.

We evaluated 119 consecutive patients with venous ulceration who were treated with the simple protocol outlined above at our institution. Six 
patients were lost to follow-up, leaving 113 for analysis of healing. These were the usual venous ulcer patients, the kind all of us see in clinical practice. About a quarter of the ulcers were recurrent ulcers. All were associated with edema and lipodermatosclerosis. Some patients had had some type of venous surgery previously. Mean ulcer diameter at presentation was $3.2 \mathrm{~cm}$. Most patients had been referred because they had been treated unsuccessfully with topical agents. Some had been treated with inadequate strength compression stockings. A few had been wearing Unna boots.

With the compressive therapy regime outlined above, we were able to heal $93 \%$ of the venous ulcers in this study. The median time of healing is admittedly long. It took a mean of $\mathbf{5 . 3}$ months to heal the patients, with some requiring more than one year to heal. Most of the patients, however, heal within 3 to 6 months. By 6 months, about $80 \%$ of the patients are healed and $87 \%$ were healed at 12 months. Compliance with the use of elastic compression stockings was very important in predicting ulcer healing; $97 \%$ of compliant patients healed, and only $55 \%$ of noncompliant patients healed their venous ulcer.

Follow-up after healing was available in 73 patients. If a patient continued to use elastic stockings after healing their venous ulcer, 5 years after healing the chance of venous ulcer recurrence was a little over $20 \%$. Noncompliant patients had 100\% recurrence by 3 years. Multivariant analysis indicated that failure to heal was associated with noncompliance with the use of elastic stockings and pretreatment ulcer duration of more than 9 months. Recurrence of ulceration after healing was associated with noncompliance with the use of elastic stockings or the development of arterial ischemia.

Other institutions have reported similar favorable results with compressive therapy. The University of North Carolina conducted a prospective study from July 1995 to July 1998. Depending on surgeon preference, three- or four-layer elastic wraps or Unna boots were utilized for compressive therapy. The investigators treated 252 venous ulcers in 217 patients. The mean age of the patients was 61 years, and the patients had the usual risk factors for venous disease. Their ulcers were slightly larger than ours in Portland. The average time that the ulcer had been present was about 7.4 months. A little over half of the patients were studied with duplex ultrasound. Only $17 \%$ of ulcers were associated with incompetent perforating veins. At 4 months, $75 \%$ of all venous ulcers were healed. At 12 months $96 \%$ were healed. Interestingly, ulcer size affected healing time, but the presence of perforator incompetence did not affect ulcer healing.

Another study from Charing Cross Hospital in London evaluated 198 legs with venous ulcers. Four-layer bandages were utilized for compressive therapy. Seventy-four percent of ulcers were healed at 6 months. The pattern of venous incompetence again did not influence healing. It didn't matter whether the patient had associated perforator incompetence or not. Healing was influenced by a previous deep venous thrombosis, increased ulcer size, and previous ulcer episodes. 
Peter will speak in some detail about the North American SEPS Registry. Briefly, this is a registry of various North American centers that have performed SEPS and contributed their data, derived from their patients, to the registry. Peter has done an excellent job of keeping track of these patients, and the contributing surgeons are to be congratulated for their efforts in this regard. In the SEPS Registry, 70\% of the limbs were treated for active ulcers. It was a safe operation. No thromboembolism, and a minimum number of wound complications occurred. Over $72 \%$ of the patients treated with SEPS also had other venous procedures. These included both superficial stripping and deep venous reconstruction. One has to ask how benefit can be ascribed to SEPS when nearly three-fourths of the patients were also treated with another procedure. I suspect the large majority had compressive therapy as well. This is truly a can of worms. You really don't know what aspect of your treatment is actually helping these patients. In addition, if you look at the percentage of ulcers healed from our study at Oregon, the study at North Carolina, and the one from Charing Cross Hospital in London, and the SEPS Registry, you find at 6 months ulcer healing was 79\% in Oregon, 75\% in North Carolina, 74\% in London, and 79\% in the SEPS Registry. I submit to you that these numbers are not much different.

Total recurrence rate of venous ulceration at 2 years was $27 \%$ at Oregon, $29 \%$ at North Carolina, and 28\% in the SEPS Registry. Again, these numbers are no different so recurrence rates with SEPS appear no different than with compressive therapy. Compressive therapy is well established, proven, simple, effective, and generally applicable to everyone. SEPS, however, is a floundering procedure, unproven, relatively complex, with unknown efficacy, and you can't use it on everybody. Peter, you have to convince us about this operation.

Dr. Kalman: That's round one; thank you, Greg. Now, it is my pleasure to introduce Dr. Peter Gloviczki to you. Peter is the newly appointed Chair of Vascular Surgery at the Mayo Clinic and Professor of Surgery at his University. He has a long-standing interest in various aspects and facets of venous disease management; as you heard this morning, he is the principal driver behind the North American SEPS registry, and today he will defend his views and biases.

Dr. Gloviczki: Good afternoon. Thank you very much, Peter, for your invitation. It is a pleasure to come to Toronto and meet our Canadian friends. It is also a great pleasure to participate in this debate with my friend, Greg Moneta, and tell you the other side of the story. The topic I was given to speak on is for the surgical treatment of venous ulcers and I am more than happy to do that. The primary surgical procedure I am going to defend is obviously the SEPS procedure (subfascial endoscopic perforator vein surgery), a minimally invasive endoscopic technique. I would like to speak up also for the surgical treatment of these patients, which is indeed necessary in addition to the conservative management as reported by the Portland group. 
The clinical problem is prevalent. We have a half million people in the United States who have active ulcerations, which is one end of the spectrum in chronic venous insufficiency. The other, milder end of the spectrum is telangiectasia and varicosed veins. The patients usually have valvular incompetence. This can involve the superficial system, perforator system, and, in some patients, the deep system. And only about $10 \%$ have significant obstructive components that actually makes this treatment more complex.

Our surgical strategy is to treat the valvular incompetence of the patient in areas that can be done safely and effectively and only go to the deeper system in case the first treatment options fail. Of all the surgical options that are available to us, high ligation and stripping of the incompetent saphenous vein is the main part of the treatment, together with avulsion of varicose veins. We have a safe and good technique to interrupt incompetent perforators in patients with advanced disease. In our patients we address the problem of perforator incompetence during the first operation. Deep vein reconstruction is done if the first operation fails. Skin grafting can be done together with any of the procedures, or independently.

The key question is, of course, is an incompetent perforating vein hemodynamically significant? Well, if it is, it deserves treatment; if it is not, it does not need treatment. The role of incompetent perforators is not completely defined, but there are definitely reasons why we should believe that perforators are, indeed, important. One analogy that Dr. Negus uses, which I think is a very good one, is to compare the incompetent perforator system to that of broken bellows. If you have a calf muscle contraction, which expresses the blood from the calf, and you have an incompetent perforator, you indeed can generate pressures around 100 to $150 \mathrm{mmHg}$ in the subcutaneous tissue. Large, incompetent perforating veins are frequently located medially, where ulcers most frequantly occur, above the ankle.

Dr. Moneta quoted a study from North Carolina, with a $17 \%$ incidence of incompetent perforators in ulcer patients. It is clearly unusual to see perforator incompetence so rarely. We compiled data of over a thousand patients with venous ulcers, reported in the literature, who underwent duplex scanning or venograph to evaluate the frequency of valvular incompetence of the different venous systems (Table 1). Ten percent of the patients had isolated superficial venous incompetence. In these patients obviously we would not do perforator vein interruption because there is no need for that. There are patients, about $5 \%$, with isolated perforator and $11 \%$ with isolated deep vein incompetence. But the group where perforator incompetence clearly plays a role is a mixed group of patients-you can see that $73 \%$ of the ulcer patients patients had duplex or venograph evidence of incompetent perforating veins. If you have incompetence in the deep veins, stripping of the saphenous system is not going to abolish systemically the perforator incompetence. If you have superficial and perforator incompetence, your perforators may or may not be interrupted with conventional stripping and avulsion techniques. There is a large group of inves- 
Table 1. Distribution of Valvular Incompetence in Patients with Venous Ulcers

\begin{tabular}{|c|c|c|c|c|c|c|}
\hline $\begin{array}{l}\text { Author, } \\
\text { Year }\end{array}$ & $\begin{array}{l}\text { Total } \\
\text { limbs } \\
\text { no. }\end{array}$ & $\begin{array}{l}\text { Sup } \\
\text { no. }(\%)\end{array}$ & $\begin{array}{l}\text { Perf } \\
\text { no. (\%) }\end{array}$ & $\begin{array}{l}\text { Deep } \\
\text { no. }(\%)\end{array}$ & $\begin{array}{l}\text { Sup + Perf } \\
\text { no. }(\%)\end{array}$ & $\begin{array}{l}\text { Sup + Perf + Deep } \\
\text { no. }(\%)\end{array}$ \\
\hline $\begin{array}{l}\text { Schanzer, } \\
1982\end{array}$ & 52 & $3(6)$ & $20(38)$ & $4(8)$ & $11(21)$ & $14(27)$ \\
\hline Negus, 1983 & 77 & $0(0)$ & $0(0)$ & $0(0)$ & $35(46)$ & $42(54)$ \\
\hline Sethia, 1984 & 60 & $0(0)$ & $5(8)$ & $20(33)$ & $17(28)$ & $18(30)$ \\
\hline $\begin{array}{l}\text { Hanrahan, } \\
1991\end{array}$ & 91 & $16(17)$ & $8(8)$ & $2(2)$ & $18(19)$ & $47(49)$ \\
\hline $\begin{array}{l}\text { van Bemmelen, } \\
1991\end{array}$ & 25 & $0(0)$ & $0(0)$ & $2(8)$ & $3(12)$ & $20(80)$ \\
\hline Darke, 1992 & 213 & $0(0)$ & $8(4)$ & $47(22)$ & $83(39)$ & $75(35)$ \\
\hline Lees, 1993 & 25 & $3(12)$ & $0(0)$ & $3(12)$ & $10(40)$ & $9(36)$ \\
\hline Shami, 1993 & 59 & $0(0)$ & $0(0)$ & $19(32)$ & $31(53)$ & $9(15)$ \\
\hline van Rij, 1994 & 120 & $48(40)$ & $6(5)$ & $10(8)$ & $31(26)$ & $25(21)$ \\
\hline Myers, 1995 & 96 & $15(16)$ & $2(2)$ & $7(8)$ & $25(26)$ & 47 (49) \\
\hline $\begin{array}{l}\text { Labropoulos, } \\
1996\end{array}$ & 120 & $26(22)$ & $1(1)$ & $5(4)$ & $23(19)$ & $65(54)$ \\
\hline $\begin{array}{l}\text { Gloviczki, } \\
1999\end{array}$ & 146 & $0(0)$ & $7(5)$ & $0(0)$ & $66(45)$ & $73(50)$ \\
\hline Total & 1084 & $111(10)$ & $57(5)$ & $119(11)$ & $353 \quad(32)$ & $444(41)$ \\
\hline
\end{tabular}

Sup $=$ superficial veins

Perf $=$ perforating veins

tigators, including our group, who believe that if there are incompetent perforators it is worthwhile to take care of them accurately; there is obviously a group of authors who do not believe that it has to be done, including the Oregon group.

Dr. Nicolaides and his colleagues studied patients with varicose veins and they found that in primary varicosity $40 \%$ had incompetent perforators; these were not ulcer patients. On the other hand, of patients who had incompetent perforators, two-thirds of them had incompetent perforators of moderate to major hemodynamic significance.

This study and several other studies confirmed that hemodynamic deterioration correlates with the severity of chronic venous insufficiency. In fact, patients with more severe chronic venous insufficiency have larger and more incompetent perforating veins.

The second question we should discuss is if SEPS is better than open perforator division. There is a prospective randomized study from Holland that found that ulcer healing was very similar between the two groups. On the other hand, the safety of SEPS over the open procedure is obvious; wound 
infection was much, much higher, and the recurrence rate at that time was equivalent. So SEPS is better than open perforator interruption.

How do we perform SEPS? I use the two-port techniques because there are several advantages: small incisions made remote from the site of ulceration, and excellent visual control with a $5-$ or $10-\mathrm{mm}$ video camera. SEPS is obviously an outpatient procedure. I regularly now use the dissecting balloon; I think it is an excellent technique to arrive immediately to a large space under the fascia. I use a thigh tourniquet because a bloodless field is an excellent operating field. The two ports that I use are moved a little bit further away from each other, so you can actually turn the second port backward and explore the entire area very effectively and avoid the "sword fighting." The interruption is either with clips and scissors or more recently I have been using the harmonic scalpel.

So what is the evidence that perforator interruption improves clinical outcome? Using the clinical scoring system designed by the American Venous Forum and a Committee of the Joint Societies, there is evidence that overall clinical symptoms in these patients improve.

What is the evidence that ulcer heals rapidly? There is quite a bit of evidence. Median healing time in the Mayo Clinic study was 35 days. In a group of 22 patients all ulcers healed in our group; we had slower healing in postthrombotic patients. We had recurrences in our own experience only in the postthrombotic patients: 5 of 12 ulcers recurred. We had no recurrence in the primary valvular incompetent group. Cumulative ulcer recurrence in the SEPS registry was $28 \%$ at 2 years; again, this has all the problems of the registry, including learning experience.

SEPS with stripping is definitely better than SEPS alone. On the other hand, those patients who had SEPS alone, those are the patients who really went through everything, all the conservative treatment, ablation of the superficial veins, so I think that in that group of patients SEPS still achieved quite good result. Even in patients who have ulcer recurrence, the clinical symptoms are much less and the ulcers are smaller. So, when we analyze the entire group of the North American Registry we found that only 8.5\% of the patient did worse. Patients who had SEPS and superficial ablation had a $22 \%$ recurrence rate. When you add individual experience, in this group of patients reported ulcer recurrence rate at about 2 years was only $10 \%$ (Table 2 ).

What is the evidence, that SEPS improves hemodynamics? Well, it is difficult to prove, reviewing the current studies in the literature. There are several studies that support the theory that SEPS, indeed, improves calf muscle function, and some studies improved venous incompetence. In the study that Dr. Rhodes did at the Mayo Clinic, we found that patients who underwent SEPS improved calf muscle function after surgery. In patients with primary valvular incompetence, calf muscle function normalized. Improvement was not significant in patients with postthrombotic syndrome, but the numbers were not very high. 
Table 2. Results of SEPS in Patients with Advanced Chronic Venous Insufficiency

\begin{tabular}{|c|c|c|c|c|c|}
\hline \multirow[b]{2}{*}{ Author, Year } & \multicolumn{2}{|c|}{$\begin{array}{l}\text { SEPS + superficial } \\
\text { ablation }\end{array}$} & \multicolumn{2}{|c|}{ SEPS alone } & \multirow[b]{2}{*}{$\begin{array}{l}\text { Follow-up } \\
\text { (months) }\end{array}$} \\
\hline & $\begin{array}{l}\text { Total } \\
\text { limbs* }\end{array}$ & $\begin{array}{l}\text { Ulcer } \\
\text { recurrence } \\
\text { no. }(\%)\end{array}$ & $\begin{array}{l}\text { Total } \\
\text { limbs* }\end{array}$ & $\begin{array}{l}\text { Ulcer } \\
\text { recurrence } \\
\text { no. }(\%)\end{array}$ & \\
\hline Jugenheimer, 1992 & - & - & 16 & $0(0)$ & 27 \\
\hline Pierik, 1995 & 4 & $0(0)$ & 34 & $1(3)$ & 44 \\
\hline Gloviczki, 1996 & 6 & $0(0)$ & 1 & $0(0)$ & 10 \\
\hline Padberg, 1996 & 11 & $0(0)$ & - & - & 16 \\
\hline Bergan, 1996 & 31 & $0(0)$ & - & - & 12 \\
\hline Wolters, 1996 & - & - & 26 & $2(8)$ & 24 \\
\hline DePalma, 1996 & 7 & $1(14)$ & 3 & $1(33)$ & 24 \\
\hline Sparks, 1997 & 12 & $0(0)$ & 7 & $0(0)$ & 9 \\
\hline Pierik, 1997 & 14 & $0(0)$ & 3 & $0(0)$ & 12 \\
\hline Rhodes, 1998 & 38 & $4(11)$ & 4 & $1(25)$ & 24 \\
\hline NASEPS, 1998 & 68 & $15(22)$ & 38 & $11(29)$ & 24 \\
\hline Murray, 1999 & 20 & $2(10)$ & 18 & $2(11)$ & 20 \\
\hline Total & 211 & $22(10)$ & 150 & $18(12)$ & 23 \\
\hline
\end{tabular}

*Class 5 limbs + Class 6 limbs after ulcer healing

In conclusion, I would say that SEPS currently performed with ablation of superficial reflux was effective to improve symptoms and to heal ulcers rapidly. Now, you can tell the patient that the ulcer will heal in 5 months, if you continue Ace bandages, but most patients after surgery heal their ulcer rapidly in a month or two. On the other hand, the main aim of the treatment should be avoiding recurrence. Recurrence or new ulcer development is significant in postthrombotic patients, and I have to admit that. I think patients who have deep venous obstruction have a poor outcome after any type of surgical procedure, and I have to agree that a prospective randomized study is justified at this point, not only to prove the need for perforator interruption but also to prove the benefit of surgical treatment over nonoperative management.

Dr. Kalman: Dr. Moneta's rebuttal, four minutes. Greg, he said some pretty nasty things about you. Would you kindly respond?

Dr. Moneta: Just business, I am sure. First of all, keep in mind it is unclear what patients are being treated with SEPS. Series that indicate very rapid healing, I believe, must be treating minimal venous ulcers. If a venous ulcer heals in only 2 to 3 weeks, it is unlikely that it was a very severe ulceration to begin with. There is a limit to how fast the fibroblasts and keratinocytes can grow and spread. SEPS is not likely to improve the regenerative activity of these cells. The important question that Peter failed to answer is "should 
SEPS should be utilized as initial therapy in the treatment of venous ulcer?" Keep in mind, Peter, as a proponent of a new therapy, has to prove the efficacy of that new therapy. An advocate of traditional therapy, such as myself, doesn't have to disprove the new therapy. Peter must provide convicing evidence that SEPS is better than conservative management. He has not done that.

There are levels of clinical evidence that apply to proving the efficacy of any therapy. The strongest clinical evidence (Level 1 ) is a large radomized trial with convincing and highly statistically significant results. The poorest level of evidence is case studies with no controls (Level 5). The SEPS literature consists virtually only of Level 5 evidence. There are only case studies; no randomized trials, no concurrent or historic controls.

It is important to realize that you don't need large numbers of patients to do a Level 1 study. Dr. Russell Hull and associates several years ago performed a Level 1 study of the treatment of proximal DVT with less than 50 patients in the study. If the difference between the outcomes evaluated in the study are dramatic, you don't need a large number of patients to prove the efficacy of one therapy over another. It is not so much that a large number of patients are required, as it is a willingness to accept the responsibility to prove that what you advocate is actually correct.

Many of you may be familiar with the Cochran Review Group. This is an international organization based in Edinburgh that systematically assesses the quality of evidence supporting various therapies. In its review process, the Cochran Review Group recently began to assess treatment of chronic venous insufficiency. Only one properly randomized clinical trial in venous disease was identified. Even this trial was not a trial for venous ulcer.

Remember, venous surgery has been advocated for venous ulceration longer than I have been alive. Despite this, no substantial evidence exists supporting operative therapy of any type in the treatment of venous ulcer. This is deplorable. Our current state of knowledge is that we can perform various operations directed toward the treatment of venous disease in a safe fashion. We by no means know whether they are efficacious. Proper trials have not been conducted. Practicing surgeons remain unconvinced.

If one looks at a list of studies reporting total recurrence rates for Linton procedures, it is obvious the Linton procedure provides results no better than those that can be obtained with conservative mangement. Dr. Linton once said that, looking back at it all, "a Linton procedure plus a stocking is as good as a stocking."

These slides depict large venous ulcers in two different patients. On the left is an ulcer that we have been treating in our clinic for over a year. The venous ulcer has probably not improved. On the right is a patient who flew out to Portland to see us in our Vascular Surgical Clinic. This patient underwent a SEPS procedure at a large, well-known, upper Midwest private institution. This large venous ulcer also is not better. 
Therapy for venous ulceration needs to improve whether it be conservative or operative. We need to achieve $100 \%$ healing of venous ulcers. We need to improve healing times and we need to ensure freedom from recurrence. For the sake of our patients, I hope that SEPS is the answer to achieving these goals. Unfortunately, however, the weight of the currently available evidence would suggest that it is not.

Dr. Kalman: Peter, it is your turn now. Do you agree with all of what Greg has said?

Dr. Gloviczki This is the reaction I expected from Dr. Moneta. He is talking about a very high healing rate and a very low recurrence rate with conservative treatment alone. But when you examine all the important studies in the literature, you will find different results (Table 3). So the study that Dr. Moneta was talking about followed 73 patients for an average of 36 months. Twenty-four of the 73 patients $(33 \%)$ developed recurrence. These numbers can only be improved if you omit noncompliant patients. One wonders, however, if that is the right way to analize the results. One study from Wisconsin, from Dr. Jonathan Town's group, reported on 90 patients; 52 ulcers recurred, with an overall recurrence rate of $58 \%$ at 10 months. When you look at 8 studies, you will find that of 488 ulcer limbs-Class 5 and Class 6 patients-252 recurred, which gives you an overall recurrence rate of $52 \%$ with medical treatment at follow-up of 27 months. So this is a very high failure rate.

Most of the patients who come to us had quite a bit of conservative management before surgical treatment. Now, what about the strictly compliant patients? In a strictly compliant group of 386 patients, reported in six studies, there were 108 recurrences, which is a $28 \%$ recurrence at 21 months after the operation. So there is a very high ulcer recurrence rate even in the very best studies in the most compliant patients.

Table 3. Results of Nonoperative Management for Advanced Chronic Venous Insufficiency

\begin{tabular}{lccc}
\hline Author, Year & Total limbs* & Recurrence no. (\%) & Follow-up (months) \\
\hline Anning, 1956 & 100 & $59(59)$ & 64 \\
Kitahara, 1982 & 59 & $8(14)$ & 12 \\
Monk, 1982 & 77 & $58(75)$ & 12 \\
Negus, 1985 & $25^{\star *}$ & $17(68)$ & - \\
Mayberry, 1991 & 73 & $24(33)$ & 24 \\
DePalma, 1992 & 11 & $11(100)$ & 10 \\
Erickson, 1995 & 90 & $52(58)$ & 28 \\
Samson, 1996 & 53 & $23(43)$ & 27 \\
Total & $\mathbf{4 8 8}$ & $\mathbf{2 5 2 ( 5 2 )}$ & \\
\hline
\end{tabular}

${ }^{*}$ Class 5 limbs + Class 6 limbs after ulcer healing

${ }^{* *}$ Class 5 limbs only 
Overall, that is what you should remember. There is a $52 \%$ ulcer recurrence after medical treatment and there is a $28 \%$ ulcer recurrence in the strictly compliant patients. So when you look at the surgical treatment group, again, if you treat superficial reflux in ulcer patients they are going to finish up with overall about $21 \%$ ulcer recurrence. The ulcer recurrence is only $10 \%$ in patients with superficial and perforator incompetence; it is higher, $30 \%$, in patients with superficial perforator and deep incompetence. If you perform SEPS with superficial reflux ablation only and you take all the studies (Table 2), the recurrence rate is really $10 \%$ and in those patients who have SEPS alone it is $12 \%$.

I have to agree with the following statement: " Patients with communicating vein incompetence in the region of venous ulcers may be best treated by interruption of incompetent communicating veins." I agree, even though this statement comes from my good friend, Greg Moneta. In his excellent chapter in Rutherford's Vascular Surgery (5th edition), Dr Moneta is telling us that indeed communicating vein incompetence should be treated with interruption of the incompetent communicating veins. There are actually surgeon who would extend the indication of perforating vein incompetence and not only to include ulcer patients, but they are so impressed by the results that they would say the best candidates for communicating vein interruption may be patients with incompetent communicating veins and early lipodermatosclerosis prior to the onset of venous ulcers. I wouldn't be so aggressive, but this is, in fact, the statement of my good friend, Dr. Moneta, who told us about a very promising procedure, SEPS. He wanted to extend the the indications. I am going to think about that.

Truly, the answer of this debate will come with the NAVUS (North American Venous Ulcer Surgery) Trial. We submitted the proposal to the National Heart Lung and Blood Institute (NHLBI) a couple of months ago I am very hopeful that it will be funded. It is going to be a study with a specific aim to identify factors leading to delayed ulcer healing and ulcer recurrence. We plan to study failure of treatment-failure to heal within 6 months, ulcer recurrence, or new ulcer formation. The secondary endpoint of the study will be the time to ulcer healing. We also want to study the rate of ulcer healing, factors associated with delayed healing or recurrence, hemodynamic improvement, quality of life, and cost efficiency.

We have to randomize 600 patients, and the main group of the study will be patients who didn't have previous operations. They will be randomized to medical and surgical arms and the surgical arm will be further randomized to superficial ablation only or to SEPS and superficial ablation. Patients who had previous stripping or who had a competent saphenous system will be randomized to medical treatment and SEPS. There would be a minimum of 2year follow-up, but it is really a 5-year study.

Until we have the results of the prospective study, we should continue to analyze our current institutional experiences, and I think we should continue to have debates like the one we have had here with Dr Moneta, or the debate 
that we had with Mr. Kevin Burnand and Dr. Hobson as moderator on the recent American Venous Forum meeting. Peter, thank you for the privilage to participate in this debate.

Dr. Kalman: We will allow a 1-minute rebuttal of the rebuttal.

Dr. Moneta: Peter is to be congratulated for his willingness to pick up the glove and attempt to conduct a randomized trial of SEPS versus conservative management in the treatment of venous ulceration. His willingness to do so indicates that, despite his rhetoric, he does truly understand that SEPS, while a promising procedure, has not been proven to be efficacious. Certainly perforator incompetence may be important in the development of venous ulceration. In addition, elimination of these perforators may improve the healing and recurrence rates of venous ulcers. It is important, however, to remember that the ultimate target organ in venous disease is the skin. When there is a long-standing damage to the skin, with extensive lipodermatosclerosis with microvascular capillary and lymphatic damage, any form of intervention is liable to be of limited efficacy. The best patients to treat with SEPS may be those patients who are developing progressive lipodermatosclerosis, but who have not as of yet sustained sufficient cutaneous damage so as to develop a venous ulcer. Viewed from this perspective, not only do we need to prove the efficacy of SEPS in healing of the existing venous ulcer, we perhaps need to consider its use in the prevention of venous ulceration. To do this we will, of course, need to understand what pathophysiologic mechanisms and what level of hemodynamic abnormalities are inevitably going to lead to lipodermatosclerosis and the risk of venous ulceration. These questions are sufficiently complex that they promise to keep us all busy for many years to come.

Dr. Gloviczki: I think the key issue is to identify patients who have hemodynamically significant incompetence of the perforating veins in any stage of chronic venous insufficiency. If we are convinced this operation adds to hemodynamic improvement, that it is effective in advanced CVI, then I think the indications of operations can be extended. There are proponents of those who say you don't need SEPS because you can take care of the perforators with avulsion or sclerotherapy. I have no argument with that. I think if you do an effective operation where you interrupt superficial and perforator incompetence in patients who have failed superficial and a failed perforator system, I think that is fine. What I am saying is, we have a technique where we can effectively interrupt perforator incompetence in addition to superficial incompetence and I think in patients who have advanced chronic venous disease, that is beneficial. But again, the NAVUS study is designed to prove this.

Moderator: I would like to open this discussion to the floor. You have the remaining 10 minutes to ask any questions or make any comment. Herb Basian has the first question.

Dr. Basian (from Audience): It has been a very worthy debate. I don't think we have all the answers. I have some questions for a change. Can you help us understand how you define the surgical, or pathological, anatomy, because I 
think one of our problems is that we are talking about not only leopards, but tigers, lions, and other cats. The experience the last numbers of years I have been exposed to this demonstrates to me that the possibility of perforator incompetence is ubiquitous; it could be medial or lateral. I think until we define the pathology it is a little like saying, it is an acute abdomen; you don't know where to make the incision without a diagnosis. You have already indicated there are the obstructive types that are going to have need for a more long-standing regimen, but I think just to use the word SEPS in the generic sense is not giving us enough detail in where to look. I would ask you how you make the diagnosis in terms of the particular areas-or is it just a blind look?

Dr. Gloviczki: No, it's not blind. We use Duplex scanning preeoperatively. If there are lateral perforators, I take care of those through small incisions, because I am concerned about peroneal nerve injury in the anterior compartment. If there is deep venous obstruction we do a venogram to see whether the patient is potentially a candidate for deep venous reconstruction or we would not remove perforating veins that are useful collateral veins.

Question from the Audience: First, what is the amount of compression prescribed for a stocking after healing of the ulcer? Second, for Dr. Gloviczki, we are seeing more publications from the American Society of Phlebology and more aggressively from France, regarding sclerotherapy under ultrasound guidance of perforators and even at the saphenofemoral junction.

Dr. Moneta: Our compression therapy is the 30 to $40 \mathrm{~mm}$ below-knee elastic stocking. We have some patients who have difficulty with stockings, and elderly patients frequently have trouble putting them on. We utilized the CircAid devices, some kind of interlocking strips of Velcro for those patients; these seem to be a little easier to apply. We use every modality that we can think of to try to keep patients using the stockings, including seeing them back frequently in the clinic so that we can encourage them to continue using some sort of compression after the healing.

Dr. Gloviczki: We use 30 to $40 \mathrm{mmHg}$ compression stockings; the compliance is about 75 to $80 \%$. There are patients who just don't like it. We had several patients who had no ulcer recurrence in spite of noncompliance. Regarding the sclerotherapy of the incompetent saphenous vein, the data I know do not support that technique. Regarding sclerotherapy for perforators, I don't know any studies with long-term follow-up. I am not against it, if it is done under duplex guidance. Being there, seeing how complex the branches of some of these perforators can be, I think that is a challenge to do a good interruption of perforating veins with sclerotherapy. But again, I have no problem with it, if they can effectively occlude it and it heals the ulcer, that's fine. There is quite a bit of evidence published in the Journal of Vascular Surgery from Drs. Wittens and Pierik showing that recurrent ulceration is associated with recurrence of persisting perforating veins.

Dr. Rutherford (from Audience): Actually, Greg, you brought this up a little bit, so I am going to ask both of you to address this particular issue. You 
mentioned it is unrealistic to expect to get long-term healing of these very badly destroyed legs, multiple, ulcerations, the whole subcutaneous tissue, musculocutaneous atrophy, scarring, misshapen legs, etc. I noticed that when you were saying that, Peter was sort of nodding a little bit, so he seems to agree that is a problem. At what point do you then take those patients and go from conservative therapy to the free-flap restoration of tissues combining with perforator interruption and other venous surgery? At the last American Venous Forum we heard a paper on this. Could you put your perspective on when you might do that, or is it indicated ever?

Dr. Moneta: First of all, there are going to be a few patients, no matter what you do and (anybody who says this is not true, I don't believe they are looking at the world realistically) you can't get the venous ulcer to heal, no matter what you do. Even if you elevate the leg straight up, 90 degrees gravity, it just won't heal. I think if you are going to treat somebody with excisional therapy and free flap, that's going to be an unusual patient because those patients are going to need to be very compliant postoperatively. So the group of patients you are talking about is small because I think the patients that you can't get healed are individuals who really are noncompliant patients. There are going to be a few others, but those are the one exception, who are very compliant and don't heal and who may be a candidate for excisional therapy, but those are probably a very few patients. Most of the patients that don't heal, they have some other problem - they just can't do it because of their mental capabilities, social situation isn't proper, their educational ability to understand is improper, and to subject those patients to a large operation and then expect them to be compliant with recommendations postoperatively isn't realistic either. So it will be a small number of patients for excisional therapy, I think.

$D$ r. Gloviczki: I fully agree that this is a spectrum of this disease. There is an endstage when tissue destruction is so extensive that it is probably in these patients that free flap should be used. This paper that Dr. Rutherford is referring to was just presented at the American Venous Forum meeting on the effectiveness of free flaps in these patients. On the other hand, we have healed quite extensive ulcerations. I am very impressed that even if the patients have extensive ulcerations, there is an intact fascia underneath. We see it very well during the SEPS procedure. It is truly amazing to me that we could always go under the ulcers, so even though it looks very extensive the destruction doesn't go all the way down to the muscle. I think if the patient otherwise is a surgical candidate and there is no gross infection, I would try to treat the underlying hemodynamic abnormality and then, depending on the size of the ulcer, do a skin graft or free flap. I think the free flap is attractive, and currently we do not use this alternative frequently enough.

Dr. Kalman: I think this was getting quite good right in the middle, but you both started saying "I agree," so it was a little disappointing at the end. Thank you both very much. Before you leave I would like to see the two of you shake hands. 
